\title{
Study and Analysis of DSR and SASR Protocols in MANET
}

\author{
Shukla Shilpa \\ Dept. of CSE, FET, MRIU \\ Faridabad, Haryana, India
}

\author{
Sharma Shelja \\ Dept. of CSE, FET, MRIU \\ Faridabad, Haryana, India
}

\author{
Kumar Suresh \\ Dept. of CSE, FET, MRIU \\ Faridabad, Haryana, India
}

\begin{abstract}
Mobile ad-hoc network is a collection of mobile nodes forming an instant network characterized by wireless links, dynamic topology and easy deployment. Random movement of nodes imposes challenges on the network resource. In this paper, we have critically analyzed the performance of DSR and its variant SASR. With the use of Sequence Number SASR, can prevent the Stale route information across the network and block their use in Route Reply. Whereas DSR can circulate the stale route information in the absence of Sequence Number, resulting in conflicting Route Discovery. Study reveals that SASR prevents spreading of Stale Route information, improving the performance as compared to DSR in terms of both Packet Delivery Ratio and Bandwidth utilization.
\end{abstract}

\section{Keywords}

Ad Hoc Networks, Route Discovery, Route maintenance, DSR, SASR, Analysis of DSR \& SASR

\section{INTRODUCTION}

Mobile means "moving" and ad-hoc means "for this purpose only or temporary" without any infrastructure. In MANET communication is done from one hop to another hop, through multihop radio relaying, without any fixed infrastructure. Mobile ad-hoc network is a collection of dynamic nodes in a network characterized by wireless links, dynamic topology and easy deployment. Routing is the process of relaying packets from source to a destination. Routing in ad-hoc network is different and challenging than wired network due to mobility of the nodes. Among two broad categories of Routing Protocols, Table- Driven Routing Protocols includes; protocols like DSDV \& WRP and On-Demand Routing Protocols include protocols like DSR, AODV \& LMR [1]. In this paper, Analysis of Route Discovery process of on-demand Routing Protocol, DSR and its variant protocol, SASR is presented. A network scenario is considered where, 9 nodes are placed randomly as shown in Figure 1. Initial node position coordinates are as follow:- $\mathrm{S}(70,170), \mathrm{A}(120,220)$, $\mathrm{B}(200,220), \mathrm{C}(250,230), \mathrm{D}(180,150), \mathrm{E}(250,95), \mathrm{F}(190,85)$, $\mathrm{G}(160,95), \mathrm{H}(120,90)$.

DSR is a simple and efficient routing protocol designed especially for use in multi-hop wireless ad-hoc networks. It is an on-demand routing

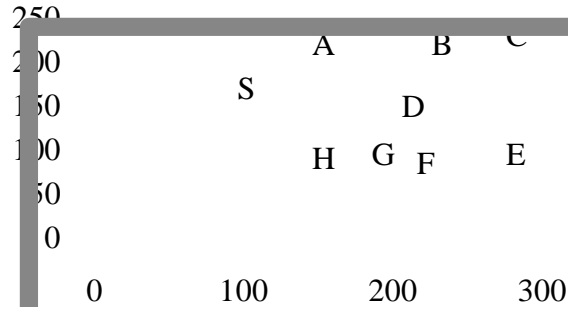

Fig 1: Initial Placement of Nodes in the Network

protocol. Like other reactive Protocol it also has two phases, Route Discovery and Route maintenance. Whenever a node finds a new path towards destination, it stores that path in its cache for future use. The disadvantage of this protocol is that the route maintenance mechanism does not repair a broken link. Stale route cache information could also result in inconsistencies during the route reconstruction phase [7].

SASR (Sequence Number Aided Source Routing) is a new reactive routing protocol, that introduces the use of Sequence Number for evaluating validity of cached routing information when source routing and route caching are used [4]. This new protocol reduces the possibility of spreading of stale route information across the network, therefore reduces the overheads involved in finding a route [4]. SASR takes advantage of Sequence Numbers to perform a passive detection of obsolete routes and block their use in Route Replies [3][4].

\section{DETAILED WORKING of DSR \& SASR}

\subsection{DSR (Dynamic Source Routing)}

DSR uses Source Routing. It is beacon-less \& hence does not require periodic hello packet (beacon) transmission. Like other On-Demand routing protocols, DSR also works in two phases: - Route Discovery and Route maintenance. Routes are discovered during Route Discovery phase and broken link information is forwarded by the corresponding node during Route Maintenance phase, without repairing a broken link. 


\subsubsection{Route Discovery Process}

DSR uses the concept of source routing. Source route is the sequence of hops between the source and the destination. Source route is defined in the header of the packet (source routing). When source node ' $S$ ' wants to send a data packet to destination node ' $D$ ', the source node ' $S$ ' first searches its cache for availability of any route to node ' $D$ '. If route to destination node ' $D$ ' is unavailable in its cache, it invokes the Route Discovery process. Source ' $S$ ' sends a Route Request (R.REQ) packet with Source address, Destination address and unique Request ID attached with the Request packet [8]. Any intermediate node upon receiving the Route Request checks Request ID, to suppress duplicate route request and searches route in its cache. If route is available then it replied back, otherwise intermediate node appends its address in route request packet and broadcast it. In this way route request packet either reaches to an intermediate node having route to the destination or at the destination itself. When Route Request (R.REQ) reaches at Destination ' $D$ ' it respond with the Route Reply (R.REP) to source 'S'. At destination node, if more than one R.REQ is received from different path; it sends RREP to all R.REQ. So, as a result of single route discovery a node can received multiple routes. For example, consider the network scenario shown in Figure 2 and 3, explaining the Route Discovery mechanism in DSR protocol. Figure 2 and 3 illustrate the Route Request and Route Reply scenario.



Fig. 2: Route Request Phase in DSR

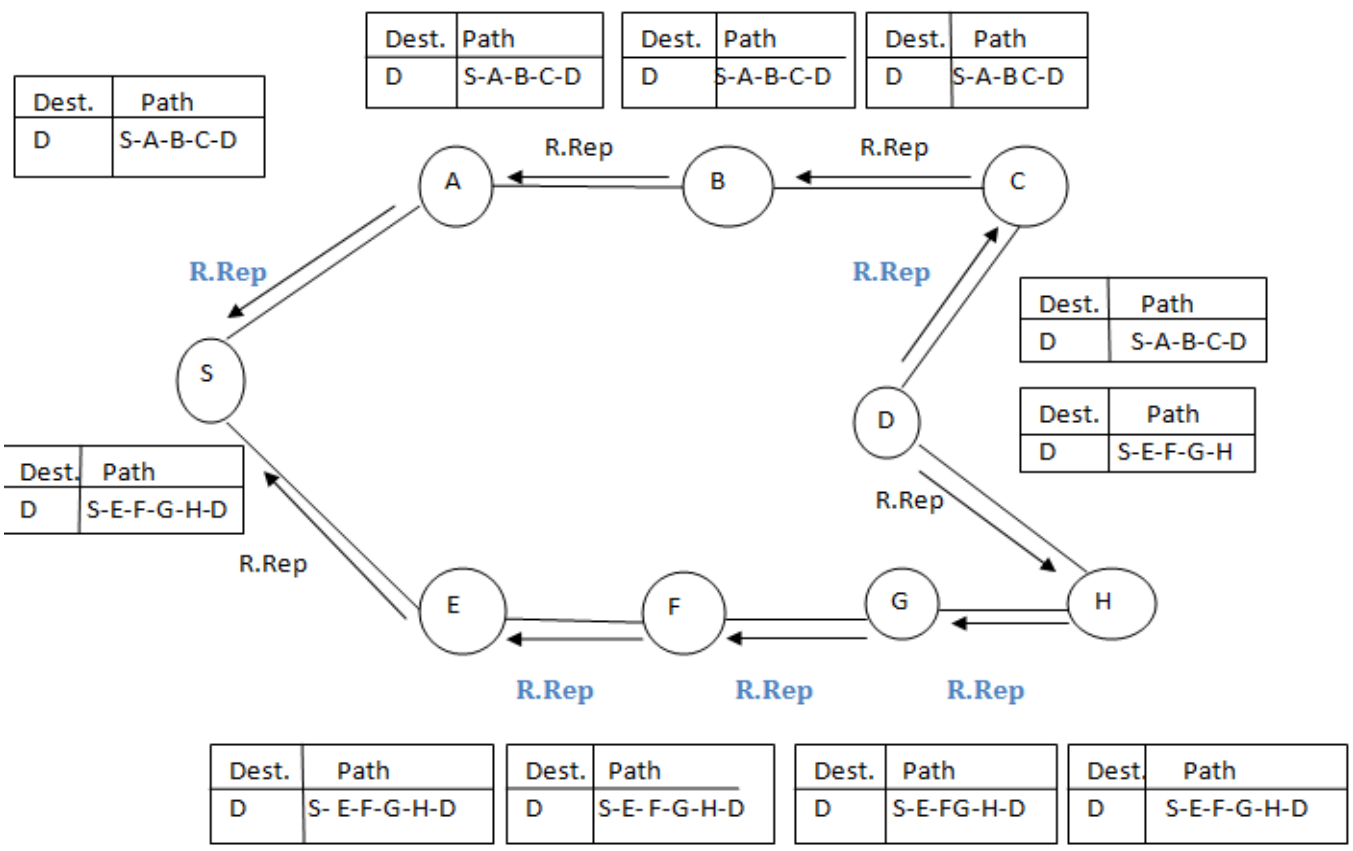

Fig. 3: Route Reply Phase in DSR 
Steps of Route Discovery Phase in Dynamic Source Routing on network scenario considered:

1. When node ' $\mathrm{S}$ ' wishes to send data to destination node ' $D$ ', it sends Route Request message to all its immediate neighbors.

2. Node ' $A$ ' \& Node ' $E$ ' check their route cache to see if the desired destination is listed.

3. Neighbor Node 'A' and Node 'E', does not found route to destination ' $\mathrm{D}$ ' in there cache. So, they append their address to Route Record of the request packet and further forward the request packet to their immediate neighbors Node 'B' \& Node 'F'.

4. In order to avoid loops and to identify duplicate request from some other path. Each neighbor check Request ID of the received Request Packet, if it has already forwarded the Route Request message with associated Request ID.

5. Finally, two Route Request reached at the destination 'D', from two different paths, as shown in the Figure 2.

6. The destination ' $D$ ' will reply both Route Request on the basis of Route Record available in the received Request Packet as shown in Figure 3.

7. Once the source receive the Route Reply message, it will send the data through the path that has the least hop count; in the above network scenario, path S-A-B-C-D.

\subsubsection{Route Maintenance Process}

Due to mobility, any active link can become inactive anytime. For spreading link break information, Route Maintenance procedure is used in DSR. There can be possibility, that link which is active now, must not be working in future. Whenever an intermediate node finds a broken link in the path from Source 'S' to Destination 'D', it sends a Route Error (R.ERR) message back to Source S [12]
When Route Error message arrives at Source 'S', it removes route to corresponding node from its cache and finds another route to Destination 'D', through Route Discovery (If no other route is available in cache). For example, consider a network scenario of 9 nodes placed randomly as shown in Figure 4 (depicts the Route Maintenance phase in DSR).

Steps of Route Maintenance Phase in Dynamic Source Routing on network scenario considered:

1. Suppose link between Node 'B' \& Node ' $C$ ' is broken as shown in Figure 4.

2. Node 'B', sends R.ERR message to its immediate neighbor Node ' $A$ ' which in turn further forward it to its next neighbor Node ' $\mathrm{S}$ ' (source node) and Node ' $\mathrm{C}$ ' forward R.ERR message to Node ' $D$ '.

3. Now, Node ' $S$ ', delete the corresponding route to destination 'D', as shown in Figure 4.

4. Now, if again data transmission is desired, Source Node ' $S$ ', check its cache for any alternate route to specific Destination ' $D$ '. In the absence of route, it again reinitiates Route Discovery.

\subsection{SASR (Sequence Number Aided Source Routing)}

SASR use Sequence Numbers for evaluating validity of cached routing information when source routing and route caching are used. SASR protocol combines source routing, caching of routes and the use of Sequence Numbers to enhance network performance in term of delivery ratio and routing overhead [3][4]. Similar to every Routing Protocol falls in category of on-demand protocols, SASR also adopt two mechanisms: - Route Discovery and Route Maintenance.

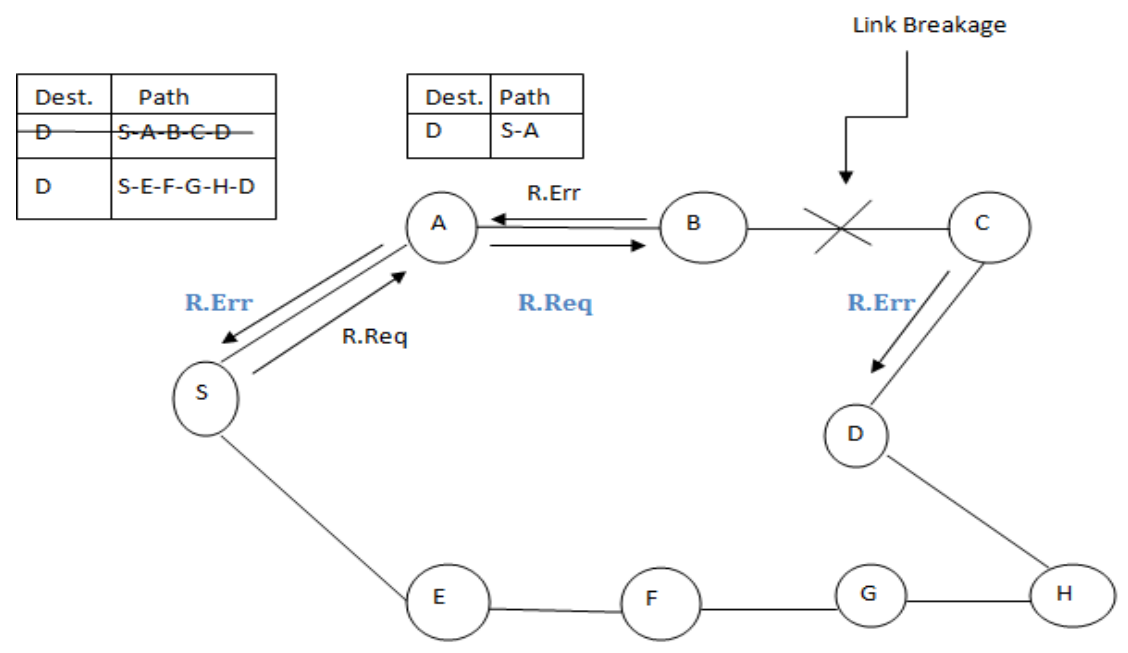

Fig. 4: Route Maintenance Phase in DSR 


\subsubsection{Route Discovery Process}

Route Discovery process includes requesting, building and establishing routes. This is referred as Route Request and Route Reply [4]. SASR is an on-demand protocol. Route Discovery is initiated only when there is need to find a route to destination. SASR avoid using source routing in the route request phase of a route discovery and limit its use in route reply, since actual caching of routes is performed at that time. SASR is an on-demand protocol.

Consider the case, where Node $n_{s}$ (address of the source node) wants to send data packet to a Node $n_{d}$ (address of the destination node). Initially, $\mathrm{n}_{\mathrm{s}}$ checks in its cache for a route to the destination. If such route does not exist Nodes increases counter $\mathrm{rn}_{\mathrm{s}}$ [3] and forward Route Request to its immediate neighbors. This packet has the structure $<\mathrm{n}_{\mathrm{s}}, \mathrm{n}_{\mathrm{prev}}, \mathrm{rn}_{\mathrm{s}}, \mathrm{sn}_{\max }$, $n_{d}>[4]$ where,

$\mathrm{n}_{\mathrm{s}}$ - Address of the source node.

$\mathrm{n}_{\text {prev }}$ - It describes the node transmitting the packet in each hop (initially $\mathrm{n}_{\text {prev }}=\mathrm{n}_{\mathrm{s}}$ ).

$\mathrm{rn}_{\mathrm{s}}$ - The source appends its request number at the time request is generated.

$\mathrm{n}_{\mathrm{d}}$ - Address of the destination node.

$\mathrm{Sn}_{\max }-$ It is the maximum Sequence Number for $\mathrm{n}_{\mathrm{d}}$ at intermediate nodes which is recorded by the packet during its travel towards destination.

$\mathrm{Sn}_{\mathrm{i}}[\mathrm{d}]-$ Sequence Number of the destination known at $\mathrm{i}$.

Each intermediate node on receiving the Route Request first checks the numbers $\left\langle\mathrm{s}, \mathrm{rn}_{\mathrm{s}}\right\rangle$. If $\mathrm{rn}_{\mathrm{s}}<=\mathrm{rn}_{\mathrm{i}}[\mathrm{s}]$ (request number of source node know at intermediate node), then the packet is discarded. Otherwise Node 'I', updates $\mathrm{rn}_{\mathrm{i}}[\mathrm{s}]$, and performs the series of action [4]:

1. If $\mathrm{Sn}_{\mathrm{i}}[\mathrm{d}]>\mathrm{Sn}_{\max } \mathrm{d}$ then the packet field $\mathrm{Sn}_{\max }$ is updated with the value $\mathrm{sn}_{\mathrm{i}}[\mathrm{d}]$.

2. Intermediate nodes $n_{i}$, adds in its cache a route entry that contain the data $\left[n_{s}, n_{\text {prev }}, \mathrm{rn}_{\mathrm{s}}\right]$, which also represent the reverse route from which the packet was received. The same route entry is used in the route reply phase, for setting up the route.
3. The packet is finally broadcasted [3].

For example, consider a network scenario where, 9 nodes are placed randomly as shown in Figure 5 (depicts the Route Request phase of SASR).

Steps of Route Request Phase in Sequence Aided Source Routing on network scenario considered:

1. When Node ' $S$ ' wishes to send data to Destination Node 'D', it send Route Request message to all its immediate neighbors as shown in Figure 5, (Node 'A' \& Node 'B') with request $\mathrm{id}=1 \& \mathrm{sn}_{\max }=100$ i.e. the last known destination Sequence Number at source 'S'.

2. On receiving Route Request, Node ' $A$ ' performs the following task:-

1. Node 'A', will maintain the request entry $\left[n_{s}, n_{\text {prev }}, r_{i d}\right]$, for constructing the reverse path. This route entry is used in the Route Reply phase.

2. Node ' $A$ ' checks if $\mathrm{rn}_{\mathrm{s}}<=\mathrm{rn}_{\mathrm{i}}[\mathrm{s}]$ then the packet is discarded, identifying incoming request as duplicate request or older request but Node ' $\mathrm{A}$ ' identify request as a fresh request and performs the following actions:-

a) Path is available in Node A's cache, as, shown in Figure 5 and figure 6. Now Sequence Number of the available route and the received Request Packet are compared as follow:-

1. If $\mathrm{Sn}_{\mathrm{i}}[\mathrm{d}]<\mathrm{Sn}_{\max } \mathrm{d}$ then the packet is forwarded to nex hop, identifying available route as stale route. Node ' $\mathrm{A}$ ' contains route to the destination ' $D$ ' but with old Sequence Number (98) and Sequence Number in the received packet is 100 . So, it forwards packet to next hop, Node 'B'.

2. If $\mathrm{Sn}_{\mathrm{i}}[\mathrm{d}]>=\mathrm{Sn}_{\max } \mathrm{d}$, then available route is replied back Node 'A' found Sequence Number of the route available, equal to the Sequence Number of the incoming request packet. So, send the available path as reply back to the source Node 'S'. 




Fig. 5: Avoidance of Stale Route Using Sequence Number

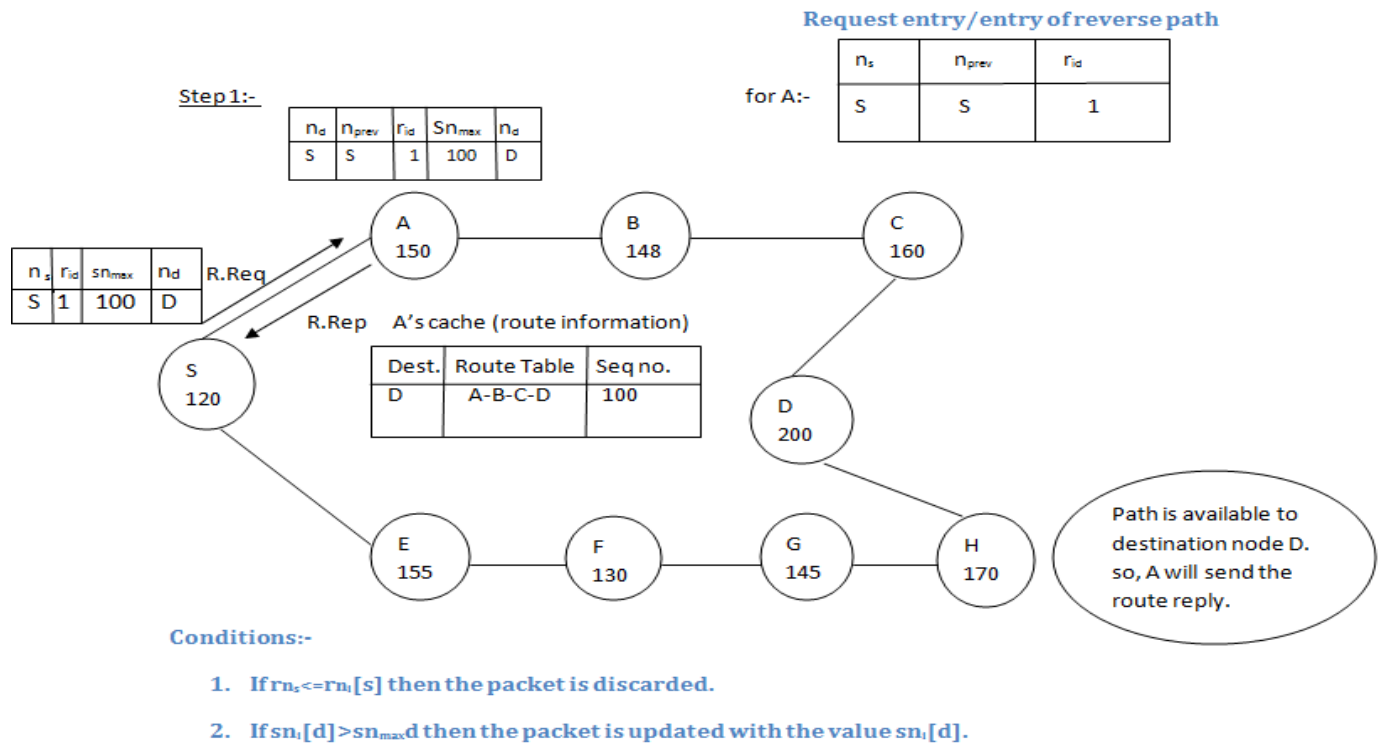

Fig. 6: Route Reply from Intermediate Node Using Sequence Number

\subsubsection{Route Reply from Destination node}

In SASR route is constructed during reply phase, whereas in DSR the discovered route has been constructed in the packet Header upon reaching the destination. The structure of the route reply packet can be represented by $\left\langle\mathrm{S}, \mathrm{rn}_{\mathrm{s}},\left\langle\mathrm{x}_{\mathrm{r}, \mathrm{j}, \mathrm{d}}\right\rangle,\left\langle\mathrm{sn}_{\mathrm{r}, \mathrm{j}, \mathrm{d}}\right\rangle, \mathrm{D}\right\rangle[$ [3]. Where,

S- Denotes the last known destination Sequence Number. $\mathrm{rn}_{\mathrm{s}-}$ Denotes the request number.

$\left\langle\mathrm{x}_{\mathrm{r}, \mathrm{j}, \mathrm{d}}\right\rangle-$ Denotes the vector which contain the addresses of the nodes consisting the $\mathrm{j}$-th route to destination $\mathrm{d}$.

$\left\langle\mathrm{sn}_{\mathrm{r}, \mathrm{j}, \mathrm{d}}\right\rangle-$ Denotes the vector of the corresponding Sequence Number.

D- Denotes the latest Sequence Number of Destination For example, consider a network scenario, under which 9 nodes have been placed randomly as shown in Figure 6 (shows route reply phase of SASR).
Steps of Route Reply Phase in Sequence Aided Source Routing on network scenario considered:

1. Upon receiving Route Request from different path the Destination Node ' $D$ ' will send the Route Reply back to its immediate neighbors as shown in Figure 7 (Node ' $\mathrm{C}$ ' \& Node ' $\mathrm{H}$ ') with $\mathrm{S}=120, \mathrm{rn}_{\mathrm{s}}=1,\left\langle\mathrm{x}_{\mathrm{r}, \mathrm{j}, \mathrm{d}}\right\rangle=\langle\mathrm{S}, \mathrm{C}-\mathrm{D}, \mathrm{D}\rangle$ for Node ' $\mathrm{C}$ ' \& $\left\langle\mathrm{x}_{\mathrm{r}, \mathrm{j}, \mathrm{d}}\right\rangle=\langle\mathrm{S}, \mathrm{H}-\mathrm{D}, \mathrm{D}\rangle$, for Node ' $\mathrm{H}$ ', $\left\langle\mathrm{sn}_{\mathrm{r}, \mathrm{j}, \mathrm{d}}\right\rangle=\langle 160-200\rangle$ for Node ' $\mathrm{C}$ ' \& $\left\langle\mathrm{sn}_{\mathrm{r}, \mathrm{j}, \mathrm{d}}\right\rangle=\langle 170-200\rangle$ for Node ' $\mathrm{H}$ ' and $\mathrm{D}=200$ (latest Sequence Number of Destination).

2. Upon receiving Route Reply, source $S$ will now have two path maintained in its cache for Destination D i.e. $<$ S-AB-C-D $>\&<$ S-E-F-G-H-D $>$. 
3. Now, Source Node 'S', will select the path, that has the lowest hop count, in this network scenario, path S-A-B-C-D is selected, as it has lowest hop count.

Now, Suppose link failure occur between S-A \& S-E due to Mobility, and corresponding routes to destination ' $D$ ' are removed from S's cache. After sometime, if Node 'S' desires to transmit data to Destination Node 'D', it will check its cache for any available route. But it does not found any, so Node 'S', again transmit Route Request Packet, to its immediate neighbors with increased Request number, as shown in Figure 8.

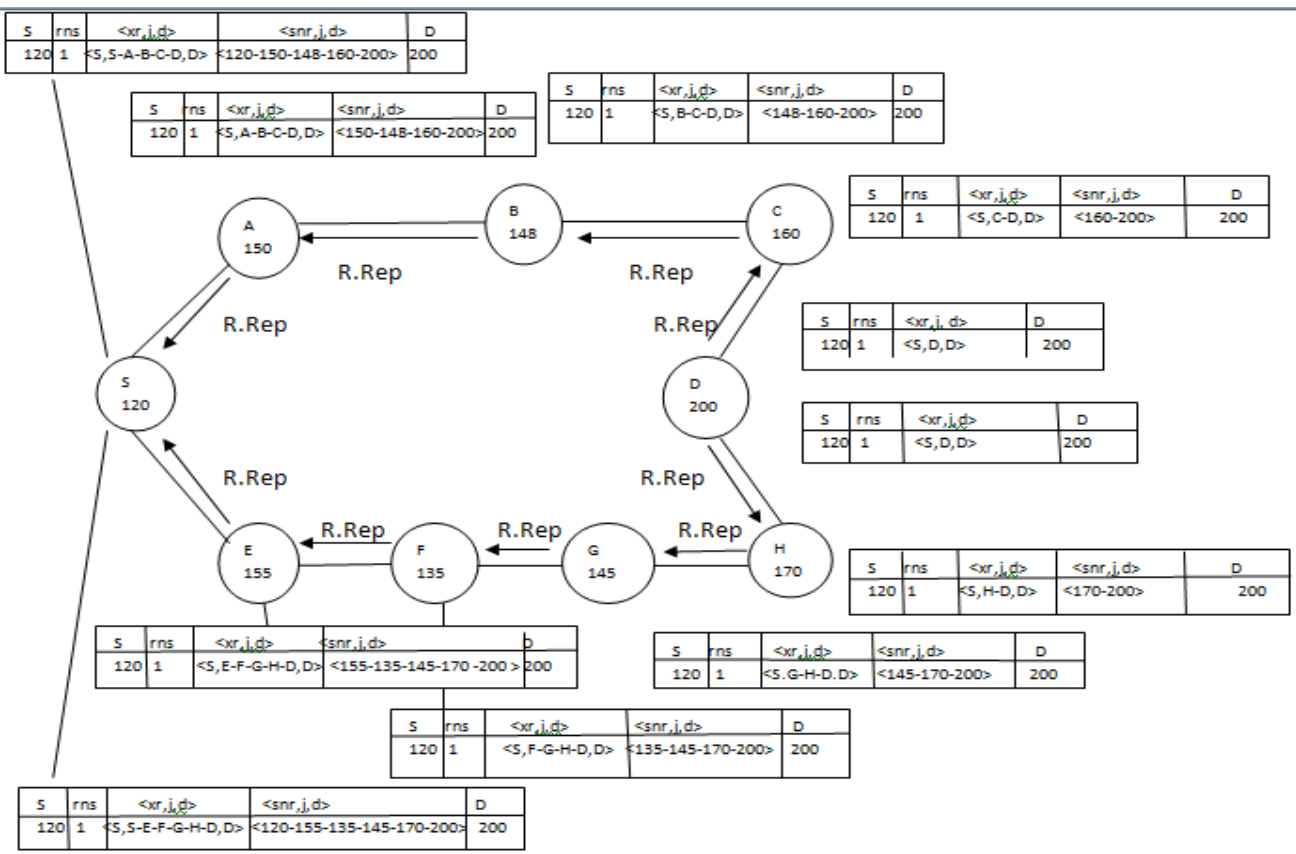

Fig. 7: Route Reply Phase in SASR

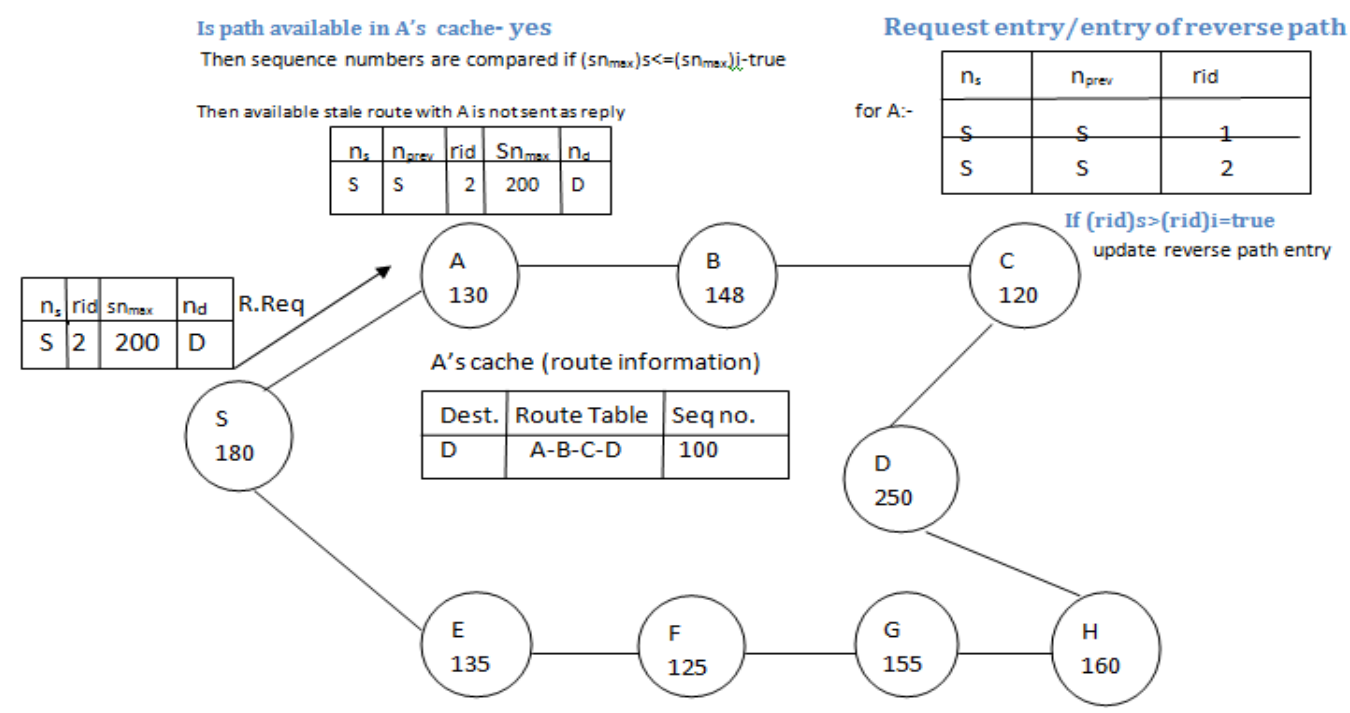

Fig. 8 Route Maintenance Phase in SASR 


\section{CONCLUSION}

Mobility is the key characteristics of MANET, any node can move from its position at any time. So, in order to prevent spreading of Stale Route information, SASR protocol uses Sequence Number associated with Request Packet. With the help of Sequence Number SASR can detect obsolete/Stale routes and block their use in Route Replies. As a result, only fresh or valid routes are replied back during Route Reply phase. Study reveals that, Packet Delivery Fraction in SASR is increased, in comparison to DSR and also Routing Overhead of SASR is less than DSR. Cache maintenance is quite difficult in DSR due to presence of stale route information while in SASR cache maintenance is much easier. In SASR there is high control traffic volume contrary to DSR. Another improvement introduced by SASR is the limited use of source routing in Route Reply phase which results in the minimization of bandwidth requirements. Table1 depicts Analysis of DSR and SASR.

Table 1: Analysis of DSR and SASR

\begin{tabular}{|l|c|l|}
\hline Parameters & DSR & SASR \\
\hline Packet Delivery Fraction & Less & More \\
\hline Routing Overhead & More & Less \\
\hline Cache Maintenance & Difficult & Easier \\
\hline Stale Route & Circulates & Prevents \\
\hline Control Traffic & Low & More \\
\hline Source Routing & $\begin{array}{c}\text { used in Route Discovery } \\
\text { \& Route Maintenance }\end{array}$ & only Route Reply \\
\hline Bandwidth Requirement & More & Less \\
\hline
\end{tabular}
Protocols: Characteristics and Comparisons" Springer, September 2004

[15] Sunil Taneja*and Ashwani Kush, "A Survey of Routing Protocols in Mobile Ad Hoc Networks", International Journal of Innovation, Management and Technology, Vol. 1, No. 3, 279-285, August 2010.
[14] Sampo Naski, "Performance Of Ad Hoc Routing

\section{REFERENCES}

[1] Asad Amir Pirzada, Chris McDonald, and Amitava Datta, "Performance Comparison Of Trust-Based Reactive Routing Protocols", IEEE June 2006.

[2] C.siva Ram Murthy, "Adhoc wireless network", $2^{\text {nd }}$ edition, Prentice Hall PTR, pp. 321-379, 2004.

[3] E.Papapetrou and F.N Pavlidou, "A Novel Approach to Source Routing for Multi-Hop AdHoc Networks", IEEE communication letter, Oct 2003.

[4] E.Papapetrou and F.N Pavlidou, "Sequence Number Aided Source Routing For Ad-Hoc Networks", Wireless personal communications, Springer 2005.

[5] Elizabeth M. Royer, University of California, Santa Barbara Chai-Keong Toh, "A Review of Current Routing Protocols for Ad Hoc Mobile Wireless Networks", IEEE Personal Communications, pp. 46-45, April 1999.

[6] Gary Breed, "Wireless Ad-Hoc network: Basic Concepts", High frequency electronics, March 2007.

[7] Google website. [Online]. Available: http://en.wikipedia.org/wiki/Dynamic_Source_Routing

[8] Huang, Chan, "Caching strategies for dynamic source routing in Mobile ad hoc networks", IEEE communication Society, March 2007.

[9] Hui Xu, Xianren Wu and J. J. Garcia-Luna-Aceves, " $A$ Unified Analysis Of Routing Protocols In MANETs", IEEE TRANSACTIONS ON COMMUNICATIONS, 911-921, March 2010.

[10] Jiang, "Performance Comparison of Three Routing Protocols For Ad Hoc Networks", IEEE Conference Publication, August 2001.

11] Loay Abusalah, Ashfaq Khokhar, Mohsen Guizani, "A Survey Of Secure Mobile Ad Hoc Routing Protocols", IEEE COMMUNICATIONS SURVEYS \& TUTORIALS, 78-93, 2008.

[12] Lou, Fang, "Predictive caching strategy for on-demand routing protocols in wireless ad hoc networks", Springer, 671-679, 2002.

[13] Mohseni, S.Hassan, R.Patel, A.Razali, "Comparative reviewstudy of reactive and proactive routing protocols in MANETs", $4^{\text {th }}$ IEEE International Conference on Digital Ecosystems and Technologies, pp. 304-309, 2010 